\title{
Development of High-Energy-Density Liquid Aerospace Fuel: A Perspective
}

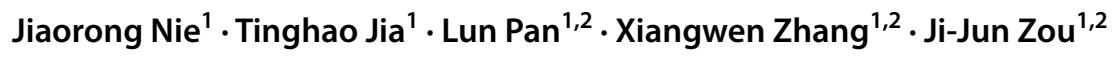

Received: 10 June 2021 / Revised: 25 June 2021 / Accepted: 28 June 2021 / Published online: 12 July 2021

(c) The Author(s) 2021

\begin{abstract}
Aerospace aircraft has significantly improved the life quality of human beings and extended the capability of space explosion since its appearance in 1903, in which liquid propellants or fuels provide the key power source. For jet fuels, its property of energy density plays an important role in determining the flight range, load, and performance of the aircraft. Therefore, the design and fabrication of high-energy-density (HED) fuels attract more and more attention from researchers all over the world. Herein, we briefly introduce the development of liquid jet fuels and HED fuels and demonstrate the future development of HED fuels. To further improve the energy density of fuel, the approaches of design and construction of multi-cyclic and stained molecule structures are proposed. To break through the density limit of hydrocarbon fuels, the addition of energetic nanoparticles in HED fuels to produce nanofluid or gelled fuels may provide a facile and effective method to significantly increase the energy density. This work provides the perspective for the development of HED fuels for advanced aircrafts.
\end{abstract}

Keyword Liquid aerospace fuel $\cdot$ High-energy-density fuel $\cdot$ Stained molecule structure $\cdot$ Nanofluid or gelled fuels

\section{Introduction}

Aerospace technology has undergone tremendous development since the first flight by Wright Brothers in 1903, which has significantly improved the life quality of human beings and extended the capability of space explosion [1]. Nowadays, aerospace vehicles mainly include commercial/military airplanes, missiles, rockets, spaceships, and satellites, which are equipped with turbine, turbofan, ramjet, or rocket engines, and liquid hydrocarbon fuels are the main energy sources for propulsion [2]. Currently, the high-performance aircrafts are aiming for high thrust-to-weight ratio, high

Jiaorong Nie and Tinghao Jia contributed to this work equally.

Lun Pan

panlun76@tju.edu.cn

Ji-Jun Zou

jj_zou@tju.edu.cn

1 Key Laboratory for Green Chemical Technology of the Ministry of Education, School of Chemical Engineering and Technology, Tianjin University, Tianjin 300072, China

2 Collaborative Innovative Center of Chemical Science and Engineering (Tianjin), Tianjin 300072, China flight speed, and long flight distance. In this case, the energy density of fuels is critically important because it determines the flight range and payload of aircraft vehicles [3]. The mostly used jet fuels, like RP-3 (directly distilled from petroleum), have a relatively low density of $0.77-0.83 \mathrm{~g} /$ $\mathrm{mL}$. Since 1950s, the high-energy-density (HED) fuels were synthesized, and considerable progress has been achieved later, especially in the past 20 years [4]. Herein, we will summarize the development of HED liquid fuels and propose the strategies to further increase their energy density.

\section{Development of Liquid HED Fuel}

For the volume-limited aircraft vehicles (especially for missiles), increasing energy density of the applied fuel helps to decrease the fuel tank volume to save more space for electronics and other components [5], or to extend the flight range and/or increase the payload without change of fuel tank [6]. The energy density of fuel is directly determined by the density and volumetric net heat of combustion (NHOC), and the latter is almost linearly positively correlated to the former (Fig. 1). Therefore, HED hydrocarbon fuel can also be named high-density fuel. The density of the widely used RP-3 is only $0.77-0.83 \mathrm{~g} / \mathrm{mL}$, 


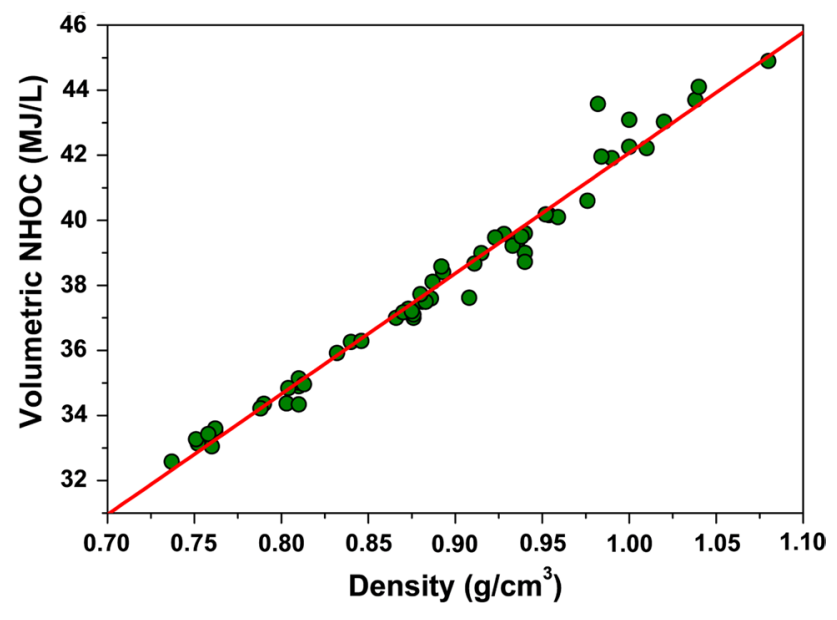

Fig. 1 Volumetric NHOC of 64 kinds of hydrocarbon fuels vs. density ( Reproduced with permission from Ref. [5]. Copyright @ 2020 )

while the density of synthetic HED fuels can reach $0.93 \mathrm{~g} /$ $\mathrm{mL}$, such as JP-10 (America), T-10 (Russia), and HD-1 (China) (with their energy density increased by ca. 16\%). Table 1 shows the composition and properties of typical HED fuels. These HED fuels all possess multi-cyclic molecule structures. The significant increase in fuel energy density can offset the slight decrease in gravimetric NHOC (caused by the decrease of hydrogen content), which is highly important to increase the flight distance of aircraft vehicles. Furthermore, liquid hydrocarbon fuels with density higher than $1.0 \mathrm{~g} / \mathrm{mL}$ have also been developed, such as RJ-5 and RJ-7. However, RJ-5 has a very high freezing point (about $0{ }^{\circ} \mathrm{C}$ ) and RJ-7 shows high viscosity ( $>400$ $\left.\mathrm{mm}^{2} / \mathrm{s} @-40^{\circ} \mathrm{C}\right)$. When these fuels are applied in aircraft vehicles, the supercooled circumstances of high altitude may freeze the fuel or increase the viscosity suddenly, which will quickly cause the shutdown of engines. Therefore, the design and synthesis of HED fuel should not only consider the property of high density, but also make sure the high performance of other physicochemical properties, like cryogenic property, flash point, thermal oxidation stability, and so on.

\section{Development of Liquid Fuels with High Energy and High Energy Density}

For the weight-limited aircraft vehicles, their take-off weight is highly restricted, such as rocket and spacecraft. In this case, the specific impulse of liquid fuel is the key property, which highly depends on the gravimetric NHOC of fuel, the matching oxidant, and the composition of combustion products [7]. Normally, the increase in gravimetric NHOC can increase the specific impulse. For example, the specific impulses of the Syntin (Russia) and quadricyclane (QC) fuels are 5-6 s higher than those of rocket kerosene (RP-1 and RP-2, using $\mathrm{O}_{2}$ as the oxidant). Different from HED fuels, the above high-energy fuels all possess the stained molecule structure to provide more combustion heat. Meanwhile, the increase in density of high-energy fuel also benefits to save more space for aircraft vehicles or extend the flight range. So, liquid fuels with both high energy and high energy density are expected to meet the demand of volumelimited and weight-limited aircraft vehicles.

As shown in Fig. 2a, for the multi-cyclic fuels with fiveor six-membered rings, the gravimetric NHOC increases linearly with $(\mathrm{H} / \mathrm{C}) / M^{0.02}(M$ is molecule weight $)$, while it decreases linearly with $(\mathrm{H} / \mathrm{C}) / M^{0.02}$ for the strained fuels with three- or four-membered rings. From Fig. 2b, the density of all fuels decreases linearly with $(\mathrm{H} / \mathrm{C}) / M^{0.19}$. The above results indicate that the multi-cyclic molecule structure with relatively low $\mathrm{H} / \mathrm{C}$ ratio (high molecule weight) and more highly strained rings will show both high-energy and HED properties. For example, the typical strained QC (with one four-membered ring and two three-membered rings) shows $18 \%$ higher density and 5-6 s higher specific impulse than those of RP-1 and RP-2.

Recently, the hydrocarbon fuels with compact polycyclic molecule structures and strained three- or four-membered rings have been synthesized, and the typical fuels and their properties are shown in Fig. 3. Obviously, the introduction of three- or four-membered rings can obtain the highly strained fuels with both high density and specific impulse. These works provide the guidance for rational design and synthesis

Table 1 Composition and properties of typical HED fuels

\begin{tabular}{|c|c|c|c|c|c|}
\hline Fuel & Composition & Density at $20{ }^{\circ} \mathrm{C}(\mathrm{g} / \mathrm{mL})$ & $\begin{array}{l}\text { Freezing } \\
\text { point }\left({ }^{\circ} \mathrm{C}\right)\end{array}$ & $\begin{array}{l}\text { Viscosity } \\
\left(\mathrm{mm}^{2} / \mathrm{s},-40\right. \\
\left.{ }^{\circ} \mathrm{C}\right)\end{array}$ & $\begin{array}{l}\text { Volumet- } \\
\text { ric NHOC } \\
(\mathrm{MJ} / \mathrm{L})\end{array}$ \\
\hline $\mathrm{RJ}-4$ & Tetrahydrodimethylcyclopentadiene & 0.930 & $<-40$ & 60 & 39.0 \\
\hline RJ-5 & endo, endo-Dihydrodinorbornadiene & 1.080 & 0 & - & 44.9 \\
\hline JP-10 (HD-01) & exo-Tetrahydrodicyclopentadiene & 0.936 & -79 & 19 & 39.6 \\
\hline $\mathrm{T}-10$ & Blend of exo- and endo-tetrahydrodicyclopentadiene & $0.942\left(15^{\circ} \mathrm{C}\right)$ & -60 & 25 & 39.7 \\
\hline RJ-7 & $\begin{array}{l}\text { Ternary blend of tetrahydrotricyclopentadiene, } \\
\text { dihydro-indanylnorbornene and JP-10 }\end{array}$ & 1.010 & - & $>400$ & 42.1 \\
\hline
\end{tabular}



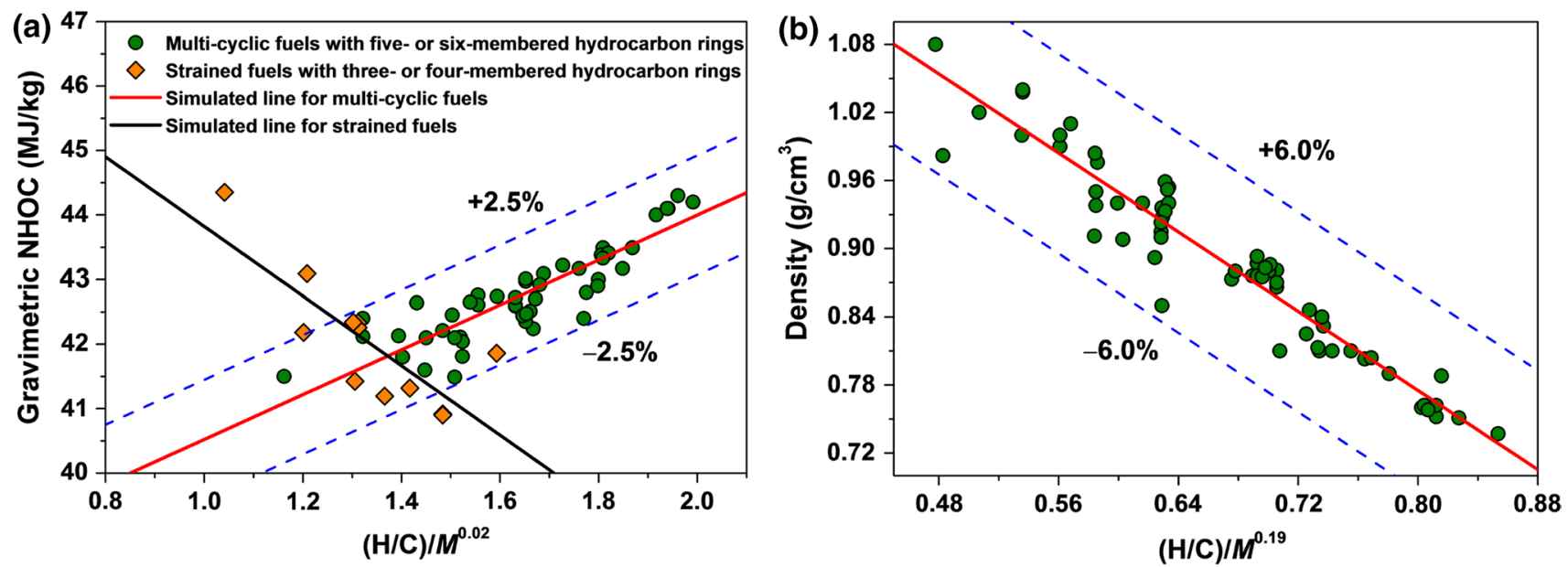

Fig. 2 a Gravimetric NHOC of 64 hydrocarbon fuels vs. (H/C)/ $M^{0.02}$ and b the densities $\left(20{ }^{\circ} \mathrm{C}\right)$ of 64 hydrocarbon fuels vs. (H/C)/M ${ }^{0.19}$ ( Reproduced with permission from Ref. [5]. Copyright () 2020)

Fig. 3 Properties of typical strained hydrocarbon fuels. Note: the specific impulse was calculated using rocket propulsion analysis software (RPA v.2.3) with $\mathrm{O}_{2}(1)$ as the oxidants, and the chamber pressure, oxidizer excess coefficient and expansion pressure ratio were $7 \mathrm{MPa}, 0.7$ and 70 , respectively

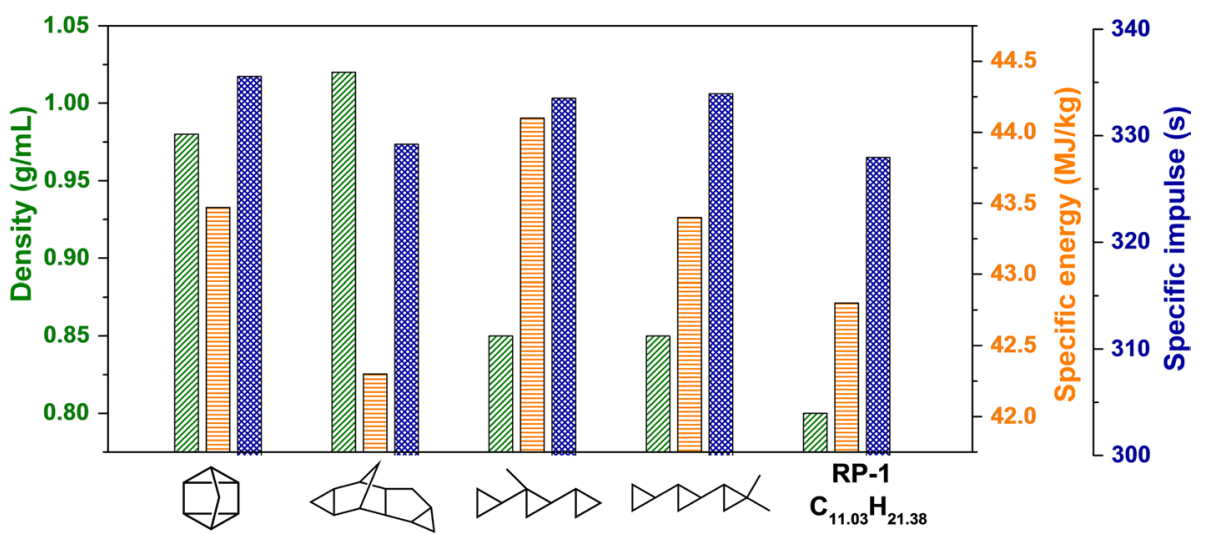

of fuels with high energy and high energy density, which are promising for the application in advanced rocket and rocketbased combined cycle engines.

\section{Energetic Nanofluid or Gelled Fuels}

Until now, impressive progress has been made in the synthesis of HED liquid fuel (including the multi-cyclic and strained fuels, derived from petroleum, coal, biomass, and so on), via thermal polymerization, isomerization, photocatalysis, and hydrogenation processes [8-12]. However, with the limitation of inherent characteristics of hydrocarbon molecules, the increase extent of energy density for HED liquid fuel is less than $30 \%$ comparing with RP-3 jet fuel.

To break through the above bottleneck, the addition of energetic nanoparticles (NPs) (such as nano-sized Al or B) in HED fuels is a very promising approach, and this class of HED suspended fuel is denoted as nanofluid fuels. Al (density of $2.7 \mathrm{~g} / \mathrm{cm}^{3}$, volumetric NHOC of $83.86 \mathrm{MJ} / \mathrm{L}$ ) and $\mathrm{B}$ (density of $2.34 \mathrm{~g} / \mathrm{cm}^{3}$, volumetric NHOC of 137.45 MJ/L) nanoparticles are the most used energetic materials to significantly increase the energy density of liquid hydrocarbon fuels [13]. Figure 4 shows the density range, gravimetric NHOC range, and volumetric NHOC range of multi-cyclic hydrocarbons, strained hydrocarbons, and nanofluid fuels. The $25 \mathrm{wt} \%$ addition of nanoAl or nano-B in HD-01 or QC HED fuels can obtain the high-performance fuel with high density of $1.1-1.25 \mathrm{~g} / \mathrm{cm}^{3}$ and high volumetric NHOC of 42.8-62.9 MJ/L. Moreover, adding $\mathrm{Al}$ or $\mathrm{B}$ nanoparticles can also shorten the ignition delay, increase the combustion efficiency and reduce the oxygen demand of fuel $[14,15]$. The combustion result of rocket engine confirms that the ignition delay time of QC containing $15 \mathrm{wt} \% \mathrm{Al}$ NPs is $26 \mathrm{~ms}$ shorter than that of pristine QC, and the volumetric specific impulse is increased by ca. 3\% [16]. Although the nanofluid fuels show very high energy density, there are still many problems that need to be solved for practical application. 


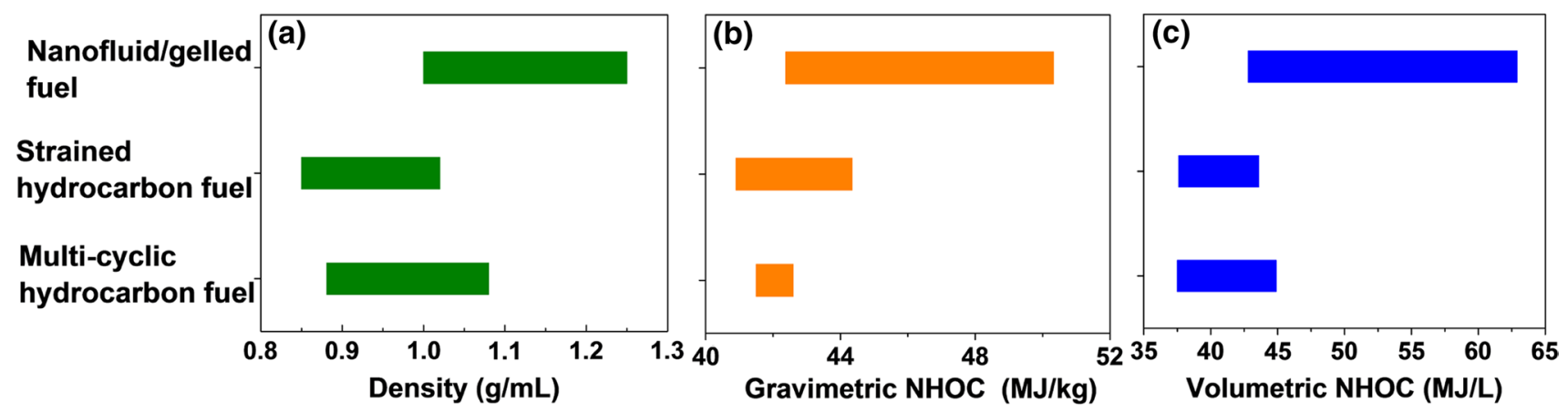

Fig. 4 a Density range, b gravimetric NHOC range, and c volumetric NHOC range of multi-cyclic hydrocarbon fuel, strained hydrocarbon fuel, and nanofluid/gelled fuels

The energetic particles are generally nanosized and easy to agglomerate and settle. Therefore, stabilizing the liquid hydrocarbon-solid NPs suspension becomes a big challenge for the synthesis, storage, and utilization of energetic nanofluid fuels. In order to obtain stable suspension nanofluid, the repulsive force among the NPs must be larger than their attractive force. Long-chain surfactant molecules can attach to the particle surface to form absorbed layer, and the overlap of the surfactant layer will produce repulsive force to overcome the van der Waals attraction, thus leading to high fuel stability. Using trioctylphosphineoxide (TOPO) as protecting ligands, the nanofluid containing $30 \mathrm{wt} . \%$ B NPs shows a good suspension property, with ca. 17\% B NPs settlement from the nanofluid fuel in $3 \mathrm{~d}$ [17].

Even though, the long-term storage stability of nanofluid fuels is still far from the demand of their practical application. The development of the fuel with phase change ability, which is prepared as a solid state with the stable state for storage and can be quickly converted into liquid for usage under the condition of shearing or heating, seems to be a promising method, like the recent developed gelled fuel system [18].

\section{Conclusions}

HED fuels attract more and more attention to promote the performance of advanced aircraft vehicles, especially for space savings and flight range extension. Different from the normal jet fuels (RP-3), HED fuels are all synthesized with very good batch stability (like JP-10). The properties of HED fuels are directly determined by their molecule structures: the multi-cyclic structures with five- or six-membered rings show high energy density and are used for volume-limited aircraft vehicles, while multi-cyclic structures with threeor four-membered rings possess high energy and specific impulse and are used for the weight-limited aircraft vehicles. However, besides the density and NHOC properties, other performance of fuels should also be concerned, like freezing point, flashing point, viscosity, during the design and synthesis of new type of advanced fuels.

Adding energetic NPs in HED fuels is expected to greatly increase the energy density, but it is very difficult to stable the high-density NPs in liquid fuels (even the effective protecting ligands have been applied), which faces many problems during the practical application (i.e., storage, transportation, atomization, and combustion). Gelling the nanofluid fuels into the non-Newtonian fluid should be a good strategy to tackle these problems.

Acknowledgements This study was financially supported by the National Natural Science Foundation of China (No. 21978200) and Scientific Research Projects of the Ministry of Education of China (No. 6141A02033522).

\section{Declarations}

Conflict of interests The authors declare that there is no conflict of interest.

Open Access This article is licensed under a Creative Commons Attribution 4.0 International License, which permits use, sharing, adaptation, distribution and reproduction in any medium or format, as long as you give appropriate credit to the original author(s) and the source, provide a link to the Creative Commons licence, and indicate if changes were made. The images or other third party material in this article are included in the article's Creative Commons licence, unless indicated otherwise in a credit line to the material. If material is not included in the article's Creative Commons licence and your intended use is not permitted by statutory regulation or exceeds the permitted use, you will need to obtain permission directly from the copyright holder. To view a copy of this licence, visit http://creativecommons.org/licenses/by/4.0/.

\section{References}

1. Edwards T (2007) Advancements in gas turbine fuels from 1943 to 2005. J Eng Gas Turbines Power 129(1):13-20 
2. Chung HS, Chen CSH, Kremer RA et al (1999) Recent developments in high-energy density liquid hydrocarbon fuels. Energy Fuels 13(3):641-649

3. Zhang XW, Pan L, Wang L et al (2018) Review on synthesis and properties of high-energy-density liquid fuels: hydrocarbons, nanofluids and energetic ionic liquids. Chem Eng Sci 180:95-125

4. Edwards T (2003) Liquid fuels and propellants for aerospace propulsion: 1903-2003. J Propuls Power 19(6):1089-1107

5. Wang XY, Jia TH, Pan L et al (2021) Review on the relationship between liquid aerospace fuel composition and their physicochemical properties. Trans Tianjin Univ 27(2):87-109

6. Muldoon JA, Harvey BG (2020) Bio-based cycloalkanes: the missing link to high-performance sustainable jet fuels. ChemSusChem 13(22):5777-5807

7. Feng R, Jia TH, Shi RH et al (2021) Computational estimation on the propulsion performance of polycyclic hydrocarbons. Chem Eng Sci. 246:116874

8. Deng Q, Nie GK, Pan L et al (2015) Highly selective self-condensation of cyclic ketones using MOF-encapsulating phosphotungstic acid for renewable high-density fuel. Green Chem 17(8):4473-4481

9. Nie GK, Shi CX, Dai YY et al (2020) Producing methylcyclopentadiene dimer and trimer based high-performance jet fuels using 5-methyl furfural. Green Chem 22(22):7765-7768

10. Li GY, Hou BL, Wang AQ et al (2019) Making JP-10 superfuel affordable with a lignocellulosic platform compound. Angewandte Chemie 131(35):12282-12286

11. Huang ZP, Zhao ZT, Zhang CF et al (2020) Enhanced photocatalytic alkane production from fatty acid decarboxylation via inhibition of radical oligomerization. Nat Catal 3(2):170-178

12. Xiao J, Zhang JX, Pan L et al (2021) Photocatalytic synthesis of high-energy-density fuel: catalysts, mechanisms, and challenges. Trans Tianjin Univ. https://doi.org/10.1007/s12209-021-00290-y

13. Ojha PK, Karmakar S (2018) Boron for liquid fuel engines-a review on synthesis, dispersion stability in liquid fuel, and combustion aspects. Prog Aerosp Sci 100:18-45

14. Guo YS, Yang YZ, Xiao J et al (2014) A novel well-dispersed nano-Ni catalyst for endothermic reaction of JP-10. Fuel 117:932-938

15. Yue L, Lu XX, Chi H et al (2014) Heat-sink enhancement of decalin and aviation kerosene prepared as nanofluids with palladium nanoparticles. Fuel 121:149-156

16. Liu Y, E X, Li ZX, et al (2019) Study on combustion performance of high-energy-density liquid fuels in rocket engine. J Propuls Technol 40(5):1169-1176 ((in Chinese))

17. E XTF, Zhi XM, Zhang YM et al (2015) Jet fuel containing ligand-protecting energetic nanoparticles: a case study of boron in JP-10. Chem Eng Sci 129:9-13
18. Cao JW, Zhang YC, Pan L et al (2020) Synthesis and characterization of gelled high-density fuels with low-molecular mass gellant. Propellants Explos Pyrotech 45(7):1018-1026

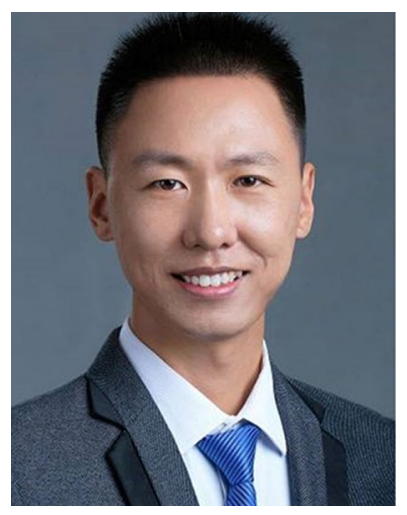

Lun Pan received his B.S. degree and $\mathrm{Ph}$.D. from the School of Chemical Engineering andTechnology, Tianjin University, China, in 2009 and 2014, respectively, and was a visiting scholar inGeorgia Institute of Technology from 2016 to 2017. Now he is an Associate Professor in TianjinUniversity, and his research interests mainly focus on the conversion and utilization of sustainablehydrogen energy, fuel processing technology and fuel science. He has authored/ coauthored 10patents and more than 50 papers, including Nat. Energy, Nat. Commun., JACS, AIChE J., CES, Adv.Mater., Nano Energy, ACS Nano, etc.

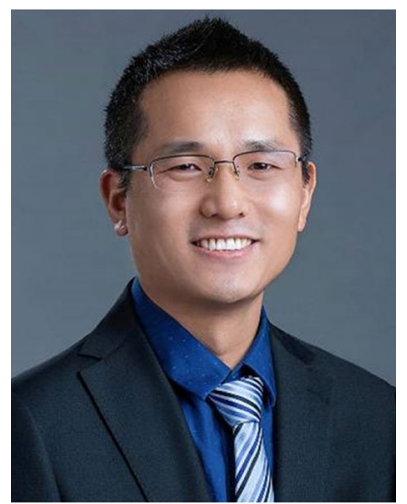

Ji-Jun Zou is the Head of Department of Chemical Technology and Chair Professor in Tianjin University, China. He received his B.S., M.S. and Ph.D. degrees in Chemical Engineering from Tianjin University in 2000, 2002 and 2005 , respectively. He was promoted as full professor of Chemical Engineering in 2013, and became Deputy Director of Key Laboratory of Advanced Fuel and Chemical Propellant of Ministry of Education in 2012. Prof. Zou has been devoted to the investigation and development of aerospace fuels for more than 10 years. He has authored/coauthored more than 150 papers and 30 patents. He has received several awards including Technological Leading Scholar of 10000 Talent project (2017), National Excellent Young Scientist by the National Natural Science Foundation of China (2012), National Excellent Doctoral Dissertation by the Ministry of Education (2008) and Peiyang Distinguished Young Scholar by Tianjin University (2009). He is also an Associate Editor of RSC Advances. 